\title{
The structure of schizotypy: relationships between neurocognitive and personality disorder features in relatives of schizophrenic patients in the UCLA Family Study
}

\author{
Keith H. Nuechterlein ${ }^{\text {a,b,* }}$, Robert F. Asarnow ${ }^{\text {a,b,c }}$, Kenneth L. Subotnik ${ }^{a}$, \\ David L. Fogelson ${ }^{a}$, Diana L. Payne ${ }^{a}$, Kenneth S. Kendler ${ }^{d}$, Michael C. Neale ${ }^{d}$, \\ Kristen C. Jacobson ${ }^{\mathrm{d}}$, Jim Mintz ${ }^{\mathrm{a}}$ \\ ${ }^{a}$ UCLA Department of Psychiatry and Biobehavioral Sciences, Los Angeles, CA, USA \\ ${ }^{\mathrm{b}}$ UCLA Department of Psychology, Los Angeles, CA, USA \\ ${ }^{\mathrm{c}}$ UCLA Mental Retardation Research Center, Los Angeles, CA, USA \\ ${ }^{\mathrm{d}}$ Departments of Psychiatry and Human Genetics, Medical College of Virginia/Virginia Commonwealth University, USA
}

Received 1 October 2001; accepted 4 October 2001

\begin{abstract}
Schizotypal personality features and certain neurocognitive deficits have been shown to aggregate in the relatives of schizophrenic patients, supporting the view that both are likely to reflect genetic contributions to liability to schizophrenia. Within the relatives of schizophrenic patients, however, the interrelationships between these potential indicators of liability to schizophrenia are not well known. Using data from the UCLA Family Study, we examine the interrelationships between personality disorder symptoms and neurocognitive functioning in nonpsychotic first-degree relatives of schizophrenic patients. Factor analyses indicate that several dimensions of schizotypy can be identified. A neurocognitive dysfunction dimension includes loadings from measures of sequential visual conceptual tracking, rapid perceptual encoding and search, and focused, sustained attention as well as the rating of odd and eccentric behavior from schizotypal personality disorder. Other aspects of schizotypal personality disorder form separate positive schizotypy and negative schizotypy dimensions. These analyses support the view that schizotypy is multidimensional in relatives of schizophrenic patients and indicate that neurocognitive deficits in perception and attention are associated with particular schizotypal personality features. (C) 2002 Elsevier Science B.V. All rights reserved.
\end{abstract}

Keywords: Neurocognitive deficits; Schizotypal personality; Schizophrenia

\footnotetext{
${ }^{*}$ Corresponding author. UCLA Department of Psychiatry and Biobehavioral Sciences, 300 UCLA Medical Plaza, Room 2240, Los Angeles, CA 90095-6968, USA. Tel.: +1-310-825-0036; fax: +1-310-206-3651.

E-mail address: keithn@ucla.edu (K.H. Nuechterlein).
}

\section{Introduction}

A series of studies have shown that schizotypal personality features and certain neurocognitive deficits aggregate in the biological relatives of schizophrenic patients as compared to the relatives of control subjects, supporting the view that both are likely to reflect genetic contributions to liability to 
schizophrenia (Asarnow et al., 1991; Braff, 1993; Kendler, 2000; Nuechterlein and Dawson, 1984; Spitzer et al., 1979; Venables, 1995). While these studies suggest the promise of both schizotypal personality features and neurocognitive deficits as tools for isolating phenotypes associated with susceptibility to schizophrenia, they have not examined the interrelationships between these two domains among relatives of schizophrenic patients through factor analytic methods. Thus, if we broaden the concept of schizotypy (Meehl, 1962; Rado, 1953) to include both neurocognitive performance deficits and clinical signs and symptoms of schizotypal personality, then this aspect of the structure of schizotypy is relatively unexplored in biological relatives of patients.

One prominent theoretical perspective that has empirical support is that schizotypal personality disorder, and probably certain other personality disorders, occur at lower thresholds on a dimension of liability than schizophrenia itself (e.g., Kendler et al., 1995; Maier et al., 1999). A possible extension of this viewpoint is that the subtle neurocognitive abnormalities that have been detected in biological relatives of schizophrenic patients may represent even lower thresholds on a single dimension of fa$\mathrm{milial} /$ genetic liability to schizophrenia, as has recently been illustrated by Maier et al. (1999) in Fig. 1. Demonstrations that certain neurocognitive abnormalities can be detected even in those relatives without any schizophrenia spectrum disorder (e.g., Green et al., 1997) are consistent with this view. The view that personality and neurocognitive features of schizotypy might fall on a single dimension of familial/genetic liability to schizophrenia is derived from

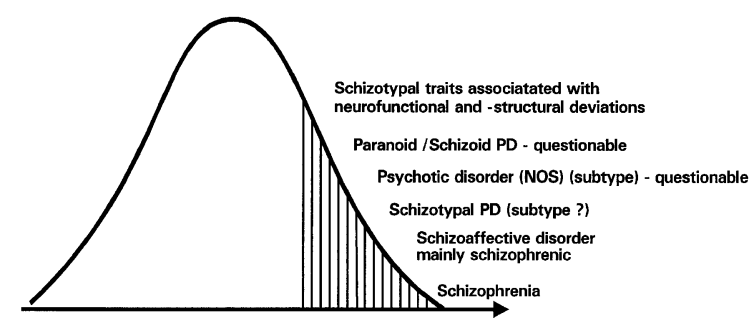

Fig. 1. Multiple threshold model for the schizophrenia spectrum (reprinted from Maier et al., 1999). the concept of liability in quantitative genetics (Falconer, 1989), which allows contributions from a number of very different sources to be viewed as additive in their contribution to susceptibility to a disease.

Another popular viewpoint that Peter Venables and others have advocated is that there are several relatively independent dimensions of schizotypal personality disorder that may be similar to dimensions of symptoms in schizophrenia (e.g., Battaglia et al., 1997; Bergman et al., 1996; Fogelson et al., 1999; Kendler et al., 1995; Raine et al., 1994; Venables and Bailes, 1994; Vollema and van den Bosch, 1995). Thus, schizotypy, as indexed by personality signs and symptoms, is likely to be a complex, multidimensional construct. From this perspective, the subtle neurocognitive abnormalities in biological relatives of schizophrenic patients could be connected to one or more of these schizotypal personality dimensions or represent entirely independent dimensions of dysfunction tied to genetic susceptibility to schizophrenia. If multiple independent dimensions are demonstrated, it would suggest that there are multiple separate dimensions of genetic contribution to susceptibility to schizophrenia, some indexed in part by neurocognitive abnormalities.

The promise of examining correlations between personality features and neurocognitive performance measures to elucidate the structure of schizotypy is illustrated in studies by Kendler et al. (1991) with a population-based sample of twins, and by Grove et al. (1991) and Vollema and Postma (in press) with firstdegree relatives of schizophrenic patients. While these studies did not combine personality features and neurocognitive performance indices in factor analytic solutions, each found that neurocognitive indices were significantly related to individual dimensions of schizotypal signs and symptoms. Kendler et al. (1991) found that attentional dysfunction (indexed by the first principal component from eight neuropsychological tests emphasizing attention) was significantly related to a negative schizotypy dimension in twins from the general population. Among relatives of schizophrenic probands, Grove et al. (1991) found that Degraded Stimulus Continuous Performance Test (DS-CPT) signal/noise discrimination scores had significant correlations with self-report Perceptual Aberration and Physical Anhedonia scores and a tendency 
toward higher correlations with the social-interpersonal component (negative schizotypy) than with the cognitive-perceptual component (positive schizotypy) of schizotypal personality disorder. In contrast, Vollema and Postma (in press), who examined three rather than two dimensions of schizotypal personality disorder symptoms, found their strongest correlations between performance on a memory-load Continuous Performance Test and a disorganization dimension. Additional studies of community samples (reviewed by Lencz et al., 1995) have not focused on the individual signs and symptoms of schizotypal personality disorder, but have demonstrated significant correlations between neurocognitive deficits and selfreport schizotypy scales.

Given some inconsistencies in the relationships found to date, a larger sample size that allows neurocognitive performance and schizotypal personality features to be examined together within the same factor solution should help to clarify the situation. A factor analytic approach with a larger sample should allow the patterns of associations and dissociations between individual schizotypal symptoms and individual neurocognitive deficits to be more reliably and clearly delineated. Furthermore, a focus on biological relatives of schizophrenic patients should increase the frequency of schizophrenia-related genes as compared to a general population sample, and may help covariation of neurocognitive performance and schizotypal features due to the influence of such genes to be detected.

Thus, the first goal of the present report was to examine schizotypal personality disorder features and selected neurocognitive performance measures in enough nonpsychotic biological relatives of schizophrenic probands to allow their interrelationships to be examined through factor analyses. Because a factor analysis of personality disorder signs and symptoms from the present project (Fogelson et al., 1999) suggested that some schizotypal personality disorder features are loaded on dimensions primarily associated with other personality disorders, the second goal was to extend the examination of the factor structure to the interrelationships between the neurocognitive performance indices and the signs and symptoms of a broader range of personality disorders that might fall within the schizophrenia spectrum.

\section{Methods}

\subsection{Participants}

The UCLA Family Study involves parallel studies of probands and relatives of three groups selected through adult probands (adult-onset schizophrenia, bipolar disorder, and community controls) (PI: K.H. Nuechterlein) and three groups selected through child probands (childhood-onset schizophrenia, attention deficit hyperactivity disorder, and community controls) (PI: R.F. Asarnow). While data collection occurred at UCLA, this report is a product of a collaboration between investigators from UCLA and the Medical College of Virginia. Written, informed consent was obtained from all adult participants after the research procedures were explained. Written assent plus written parental consent were obtained for all participants who were minors. This report includes 313 nonpsychotic biological relatives of probands diagnosed with DSM-III-R schizophrenia or schizoaffective disorder, depressed type. Relatives of adultonset schizophrenia probands $(n=228)$ and of childhood-onset schizophrenia probands $(n=85)$ who were 15 years of age or greater at the time of assessment were combined for these analyses.

The procedures for selection of the probands have been described in detail elsewhere for adult-onset schizophrenia (Nuechterlein et al., 1992; Subotnik et al., 1997) and childhood-onset schizophrenia (Asarnow et al., 2001). Briefly, the adult-onset schizophrenia probands were recruited from a study of schizophrenia patients with a recent adult onset of psychosis, Developmental Processes in Schizophrenic Disorders (Nuechterlein et al., 1992), which drew its sample from consecutive admissions to four large public hospitals in Los Angeles and from outpatient referrals to the Aftercare Research Program of the UCLA Neuropsychiatric Institute. The childhoodonset schizophrenia probands (onset before age 13) were recruited from the UCLA Neuropsychiatric Hospital, residential treatment programs, and facilities for children with severe emotional disturbances in the Los Angeles County Department of Mental Health and the Los Angeles Unified School District. Individuals with neurological disorders, or with substance abuse that obscured the source of psychotic symptoms, were excluded. 
The direct participants in this report included 170 parents, 124 full siblings, and 19 half-siblings of 147 adult-onset and childhood-onset schizophrenia probands. Half-siblings were included to maximize sample size, given that they would also have an increased frequency of schizophrenia-related genes relative to population base rates. This sample represents all firstdegree relatives and half-siblings of the probands who were at least 15 years of age, who had never experienced a psychotic disorder, and who participated in and had valid data from the psychiatric interviews and neurocognitive assessments described below. Relatives less than 15 years of age at assessment $(n=25)$ were excluded from this report because the validity of personality disorder features below this age is uncertain. Relatives with psychotic disorders $(n=23)$ were excluded from these analyses to eliminate effects of alterations in neurocognitive functioning after psychosis onset and to avoid the difficulties of assessing premorbid personality disorder in individuals who have developed full-blown psychotic disorders. In addition, nine relatives were excluded from these analyses due to poor visual acuity that invalidated the visual neurocognitive testing, 36 for other reasons that invalidated one or more neurocognitive scores as indices of schizotypy (e.g., significant neurological disorder, current use of medication with strong sedative or stimulant effects, equipment failure), 12 due to inadequate English fluency to provide valid WAIS vocabulary scores, and two because insufficient information was available to rate schizotypal personality disorder signs and symptoms validly.

The 313 relatives included 143 males and 170 females, had a mean age of 39.2 years $($ S.D. $=14.7)$, and a mean educational level of 13.8 years (S.D. $=2.6$ ). The ethnic/racial composition was $70 \%$ non-Hispanic Caucasian, 12\% Hispanic, 7\% African-American, 4\% Asian, 1\% American Indian or Alaskan, and 6\% "other" or mixed.

\subsection{Procedures}

\subsubsection{Diagnostic assessment}

All participants were directly administered the Structured Clinical Interview for DSM-III-R Personality Disorders (SCID-II; Spitzer et al., 1990). Interrater reliability for symptom dimensions was shown to be adequate (Fogelson et al., 1991). Whenever possi- ble, two adult members of each family, usually the parents of the proband, were also administered a SCID-II version that was adapted to elicit personality disorder information regarding other family members. The information from self-reports and from other family members were then reviewed by project staff, blind to diagnosis of proband, to reach consensus ratings of signs and symptoms of personality disorder. Given the issues concerning the degree to which individuals lack insight into their own personality disorder features, this combination of information was used to enhance validity of final ratings. The signs and symptoms of four personality disorders believed to be relevant to the schizophrenia spectrum were rated (schizotypal, paranoid, schizoid, and avoidant), as well as the signs and symptoms of borderline personality disorder.

\subsubsection{Neurocognitive assessment}

Three neurocognitive measures that have detected subtle deficits in sustained, focused attention, early perceptual processes, and sequential visual tracking in relatives of schizophrenia patients (Asarnow et al., 1991; Braff, 1993; Kremen et al., 1994; Nuechterlein, 1991) were administered to each participant, as described below. Elsewhere, the literature-supporting selection of these measures has been summarized (Nuechterlein et al., 1998). The WAIS Vocabulary subtest (Wechsler, 1955) was included to assess general verbal ability level.

The Degraded Stimulus Continuous Performance Test (DS-CPT), a measure of sustained, focused visual attention, involves detection of each occurrence of a highly blurred target digit within a quasi-random series of blurred, single-digit stimuli presented at $40 \mathrm{~ms}$ each at a pace of $1 / \mathrm{s}$ over an 8 -min period (Nuechterlein et al., 1983). Blurring (degradation) of stimuli enhances the role of early perceptual encoding in performance. Details of stimulus characteristics and projector equipment were given in Nuechterlein et al. (1986). The signal/noise discrimination index, $d^{\prime}$, from signal detection theory was the dependent variable.

The Forced-Choice Span of Apprehension Task (Span), a measure of rapid perceptual encoding and efficient covert visual search, involves detection of target letters ( $\mathrm{T}$ or F) within briefly presented arrays of other letters (Asarnow and MacCrimmon, 1981; Asarnow et al., 1991). The partial-report, forced-choice 
format limits the role of short-term memory and enhances the role of early encoding and search processes. Stimulus characteristics and projector equipment for this version were described in Nuechterlein et al. (1986). The accuracy of target detection in 10letter arrays was the dependent variable.

The Trail Making Test, Part B (Trails B), a measure of sequential visual conceptual tracking, involves connecting numerals and letters on a sheet of paper as quickly as possible (Reitan, 1958). The need to alternate between a number series and a letter series ( $1, A, 2, B, 3, C$, etc.) entails demands on maintenance of task set over time, working memory for position in the two sequences, and organized visual scanning. An adolescent version was used to accommodate the age range of subjects in this study. The number of seconds required to complete Part B was used as the dependent variable. A reciprocal transformation was used to normalize the distribution of scores, resulting in a variable reflecting Trails B speed.

Given the wide range of ages included in this sample (15-75 years), the neurocognitive performance measures were corrected for normal aging. A model of normal age effects was derived for each variable from the community control subjects who had no DSM-III-R Axis I or Axis II diagnoses. This sample of 277 individuals was part of a demographically matched community sample and had a distribution of age, gender, and educational level that was similar to that of the relatives of schizophrenic pro- bands. The age-corrected neurocognitive score was the residual after the model of normal age effects was applied. WAIS Vocabulary scores were age-corrected using the tables in Wechsler (1955). These age corrections serve to limit the chances that observed correlations with personality signs and symptoms are due to any joint relationship to normal aging.

\subsection{Factor analytic procedures}

The MPLUS program (Version 1.04) from Muthén and Muthén (2001) was used with unweighed least squares estimation to complete these analyses. This program is designed to combine ordered categorical variables with non-normal distributions (personality disorder features) and continuous variables (neurocognitive performance scores) in the same analysis. Exploratory factor analysis, rather than confirmatory factor analysis, was used because the interrelationships of the neurocognitive measures and the personality disorder signs and symptoms were not sufficiently explored in prior research to allow a factor structure for their combination to be clearly hypothesized.

\section{Results}

An initial exploratory factor analysis examined the interrelationships between the nine signs and symptoms of DSM-III-R schizotypal personality disorder,

Table 1

Varimax factor loadings for three-factor solution: schizotypal personality disorder and neurocognitive variables

\begin{tabular}{lccc}
\hline Variable & Negative schizotypy & Positive schizotypy & Cognitive disorganization \\
\hline Trails B speed & -0.03 & -0.03 & -0.89 \\
Span 10-letter target detection & 0.01 & 0.09 & -0.38 \\
DS-CPT d $d^{\prime}$ & -0.03 & -0.05 & -0.31 \\
WAIS Vocabulary & -0.12 & 0.00 & -0.17 \\
SPD1: ideas of reference & 0.70 & 0.58 & -0.12 \\
SPD2: social anxiety & 0.51 & 0.21 & -0.01 \\
SPD3: odd beliefs or magical thinking & 0.19 & 0.77 & 0.01 \\
SPD4: unusual perceptual experiences & 0.06 & 0.82 & 0.02 \\
SPD5: odd or eccentric behavior & 0.76 & 0.15 & 0.61 \\
SPD6: no close friends & 0.42 & 0.04 & 0.24 \\
SPD7: odd speech & 0.75 & -0.03 & 0.07 \\
SPD8: inappropriate/constricted affect & 0.75 & -0.56 & 0.04 \\
SPD9: suspiciousness, paranoid ideation & 0.49 & 0.27 & 0.07 \\
Eigenvalue & 3.71 & 2.19 & 1.62
\end{tabular}

Note: SPD = schizotypal personality disorder; numbers following SPD refer to the number of the symptom or sign in DSM-III-R. 
Table 2

Varimax factor loadings for seven-factor solution: neurocognitive variables and signs and symptoms from five DSM-III-R personality disorders $^{\mathrm{a}}$

\begin{tabular}{lc}
\hline & Loading \\
\hline Borderline symptoms & \\
Affective instability & 0.80 \\
Unstable, intense relationships & 0.79 \\
Impulsivity & 0.72 \\
Identity disturbance & 0.71 \\
Inappropriate, intense anger & 0.69 \\
Feelings of emptiness or boredom & 0.68 \\
Recurrent suicidal threats or behavior & 0.64 \\
Frantic efforts to avoid abandonment & 0.60 \\
Easily slighted, vindictive & 0.59 \\
Odd or eccentric behavior & 0.45 \\
Questions fidelity without justification & 0.42 \\
Ideas of reference & 0.40 \\
& \\
Schizoid symptoms & \\
Little desire for close relationships & 0.95 \\
Rarely has strong feelings & 0.81 \\
Chooses solitary activities & 0.75 \\
Little desire for sexual relationships & 0.60 \\
Indifferent to praise and criticism & 0.56 \\
Avoids interpersonal contact & 0.51 \\
Constricted affect & 0.50 \\
No close friends & 0.47 \\
Does not take initiative in relationships & 0.41
\end{tabular}

Avoidant symptoms

Social anxiety

Reticent in social situations

Fears being embarrassed by anxiety

Avoids interpersonal contact

Exaggerates dangers and risks

Does not take initiative in relationships

Feelings easily hurt

\section{Negative schizotypy}

Inappropriate or constricted affect

Constricted affect

Odd or eccentric behavior

Odd speech

\section{Paranoid symptoms}

Suspiciousness or paranoid ideation

Questions loyalty of friends

Bears grudges, unforgiving

Reluctant to confide

Hidden threatening meanings

Expects to be exploited or harmed

Easily slighted, vindictive
Table 2 (continued)

\begin{tabular}{lr}
\hline & Loading \\
\hline Positive schizotypy & \\
Unusual perceptual experiences & 0.96 \\
Odd ideas or magical beliefs & 0.66 \\
Ideas of reference & 0.47 \\
& \\
Cognitive disorganization & -0.71 \\
Trails B speed & 0.58 \\
Odd or eccentric behavior & -0.44 \\
Span 10-letter target detection & \\
\multicolumn{2}{c}{ a Only items with loadings with magnitude of at least 0.40 are } \\
listed.
\end{tabular}

the three neurocognitive variables, and the WAIS vocabulary score. The three-factor solution was chosen because it had an easily interpretable simple structure and because examination of the scree plot for unrotated factors supported a cutoff after three factors. Eigenvalues dropped sharply after three factors (eigenvalues were $3.72,2.19,1.62$, and then, 1.16 and 1.12 for the first five factors). Varimax and promax rotations yielded very similar loadings; the loadings on the orthogonal factors are presented in Table 1.

Based on an examination of the items loading highly on the factors and consideration of prior factor analyses of schizotypal personality disorder items, we labeled the first two factors as negative schizotypy and positive schizotypy. Because the three neurocognitive measures all loaded primarily on the third factor, combined with the schizotypal personality disorder sign, odd or eccentric behavior or appearance, we labeled this factor as Cognitive Disorganization. Trails B speed, which reflects the efficient combination of maintenance of task set, working memory, and visual tracking, was the item that loaded most highly on this third factor.

To determine how these three factors fit into the context of dimensions from other personality dimensions, including those that may represent additional schizophrenia spectrum disorders, we completed a second exploratory factor analysis in which the signs and symptoms from paranoid, schizoid, avoidant, and borderline personality disorders were added. A sevenfactor solution was selected based on examination of the scree plot for unrotated factors and on its ease of interpretation. Varimax and promax rotations again yielded very similar loadings. Table 2 presents the loadings on the orthogonal factors. 
This seven-factor solution adds separate factors for borderline, schizoid, avoidant, and paranoid dimensions, with the DSM-III-R items for each of these personality disorders falling coherently on a discrete and highly interpretable factor. The three factors from the initial factor analysis are relatively unaffected by the inclusion of items from these other personality disorders in this second analysis.

\section{Discussion}

In the biological relatives of schizophrenic patients, three neurocognitive measures of potential vulnerability factors for schizophrenia and a sign of schizotypal personality disorder, odd or eccentric behavior or appearance, fall on a single factor. Trails B speed is the item in the present analysis that loads most highly on this factor, with lesser loadings from the indices reflecting target discrimination accuracy within the span of apprehension task and the Degraded-Stimulus CPT. We labeled this factor as cognitive disorganization to recognize its core neurocognitive nature and to suggest that cognitive disorganization contributes to the form of odd and peculiar behavior or appearance that loads on this factor. This factor appears be related to the disorganized factor of Raine et al. (1994), although odd speech did not load on the present factor.

Another schizotypy factor that we isolated among relatives of schizophrenia patients, negative schizotypy, is similar to the oddness factor of Battaglia et al. (1997) but also includes somewhat lower loadings from the items of their interpersonal factor. It also shares many features with the interpersonal factor reported by Raine et al. (1994) and the negative symptom schizotypy factor of Kendler et al. (1991).

A third schizotypy factor that emerged, positive schizotypy, receives high loadings from symptoms that appear to be less severe variants of a dimension of reality distortion that has delusions and hallucinations at its severe end. It is very consistent with the positive schizotypy factor of Kendler et al. (1995) and the cognitive-perceptual schizotypy factors of Raine et al. (1994) and Battaglia et al. (1997). We prefer the label positive schizotypy over cognitive-perceptual factor to distinguish this dimension from the one on which the cognitive performance indices fall.
The schizotypal sign, odd or peculiar behavior, that loads with the three neurocognitive indices is noteworthy in that it has been found to fall on a dimension of schizotypal personality disorder that was among the best discriminators of relatives of schizophrenic patients and community controls in a large-scale population-based study (Kendler et al., 1995). The availability of continuous neurocognitive variables to supplement the schizotypal sign of odd behavior should allow measurement of the Cognitive Disorganization schizotypy dimension in a way that is much more sensitive to quantitative variations in susceptibility to schizophrenia than is a schizotypal personality sign alone. Such neurocognitive performance indices may also help to identify the neural substrate of this dimension of schizotypy, as these neurocognitive tasks can be examined within neuroimaging activation paradigms to elicit cognitive deficits related to susceptibility to schizophrenia (e.g., Buchsbaum et al., 1990; Strandburg et al., 1991). Inclusion of a wider range of neurocognitive measures, including working memory and secondary memory assessments, in future studies would help to determine whether cognitive deficits contribute to just one or to several dimensions of schizotypy.

Also noteworthy is that the schizotypal sign of odd and peculiar behavior or appearance loads on several different factors, and is thus not factorially pure. Examination of the individual behaviors that were rated positive on this item within this sample suggests a possible reason for this phenomenon. Some of the behaviors appear to reflect a lack of interest in grooming and dress, and would fit well with other behaviors typically considered to be aspects of negative schizotypy. Other behaviors seem to reflect a more active process of behavioral disorganization, in which different aspects of the individual's behavior or appearance are incongruous or asynchronous despite what appear to be very active, motivated efforts. Thus, future studies might benefit from trying to separate these two contributors to this schizotypal personality disorder item.

The observation that a process of behavioral disorganization might contribute to some instances of odd and peculiar behavior or appearance may also help to connect this schizotypal sign to underlying cognitive processes. The three neurocognitive measures in this study involve speeded performance requir- 
ing rapid perceptual encoding and discrimination of predesignated targets (DS-CPT, Span) and rapid, organized visual search for predesignated stimuli (Span, Trails B). Trails B speed, which was most strongly related to the odd behavior item, also involves additional elements of working memory for two stimulus sequences to guide visual search efficiently. Thus, all three, and particularly the Trails B measure, may partially reflect the effectiveness of goal-directed searches for predesignated target stimuli. Subtle impairments in such goal-directed cognitive processes may also contribute to behavioral disorganization at the molar level, as reflected in the observations of odd behavior. An alternative, and compatible, conception has emphasized failures in cognitive inhibition (Lencz et al., 1995) or more specifically in inhibition of irrelevant responses (Vollema and Postma, in press) as a factor contributing to schizotypy or specifically to disorganization. Cognitive tasks that allow more differentiated parsing of different components of processing will be needed in future studies to shed light on the distinct cognitive processes contributing to odd behavior.

Possible limitations of the present analyses include the use of exploratory rather than confirmatory factor analysis and the absence of correction for correlated observations. Confirmatory factor analysis has the advantages of allowing tests for a priori hypotheses about the number of factors and about their composition. We believe that exploratory factor analyses are appropriate at this initial point and did not find that different rotation procedures altered the basic findings. However, confirmatory factor analysis would be a valuable step in future research. Use of a factor analytic procedure that allows correction for correlated observations (multiple observations per family), while still allowing for the inclusion of both ordered categorical and continuous variables, would also refine the present analyses.

The present data from nonpsychotic relatives of schizophrenic patients provide further evidence favoring a multidimensional model of schizotypy. This multidimensionality is likely to imply that several different factors contribute to typical cases of schizotypal personality disorder, with the importance of any given factor varying from case to case. As suggested by Venables (1995), the forms of schizophrenia that occur in individuals may involve the development of different patterns of abnormality across the dimensions of schizotypy that continue in a parallel pattern after onset of schizophrenia. If so, we should consider whether the familial/genetic influences on these different schizotypy dimensions may also be partially separable. Our present data from nonpsychotic relatives of schizophrenic patients, while not testing this hypothesis directly, would be consistent with this possibility.

\section{Acknowledgements}

This research was supported in part by NIMH grants MH49716, MH45112, MH37705, MH30792, MH14584, and MH30911 and by a grant from the John D. and Catherine T. MacArthur Foundation Research Network on Risk and Protective Factors in the Major Mental Disorders. We acknowledge the very helpful statistical services of Sun Hwang, M.S., M.P.H. The authors also wish to thank Dr. Bengt Muthén for the statistical consultation and the staff and participants of the UCLA Family Study for their essential contributions to this effort. This article benefitted from the insightful comments of Drs. Bruce Wexler and Meinte Vollema, following an oral presentation of its contents at the NATO Advanced Research Workshop.

\section{References}

Asarnow, R.F., MacCrimmon, D.J., 1981. Span of apprehension deficits during the post-psychotic stages of schizophrenia: a replication and extension. Archives of General Psychiatry 38, 1006-1011.

Asarnow, R.F., Granholm, E.L., Sherman, T., 1991. Span of apprehension in schizophrenia. In: Steinhauer, S., Gruzelier, J., Zubin, J. (Eds.), Handbook of Schizophrenia. Neuropsychology, Psychophysiology and Information Processing, vol. 5. Elsevier, Amsterdam, pp. 335-370.

Asarnow, R.F., Nuechterlein, K.H., Fogelson, D., Subotnik, K.L., Payne, D.A., Russell, A.T., Asamen, J., Kuppinger, H., Kendler, K.S., 2001. Schizophrenia and schizophrenia-spectrum personality disorders in the first-degree relatives of children with schizophrenia: the UCLA Family Study. Archives of General Psychiatry $58,581-588$.

Battaglia, M., Cavallini, M.C., Macciardi, F., Bellodi, L., 1997. The structure of DSM-III-R schizotypal personality disorder diagnosed by direct interviews. Schizophrenia Bulletin 23, 83-92.

Bergman, A.J., Harvey, P.D., Mitropoulou, V., Aronson, A., Mard- 
er, D., Silverman, J., Trestman, R., Siever, L.J., 1996. The factor structure of schizotypal symptoms in a clinical population. Schizophrenia Bulletin 22, 501-509.

Braff, D., 1993. Information processing and attention dysfunctions in schizophrenia. Schizophrenia Bulletin 19, 233-259.

Buchsbaum, M.S., Nuechterlein, K.H., Haier, R.J., Wu, J.C., Sicotte, N.L., Hazlett, E.A., Asarnow, R.A., Potkin, S., Guich, S., 1990. Glucose metabolic rate in normals and schizophrenics during the continuous performance test assessed by positron emission tomography. British Journal of Psychiatry 156, 216227.

Falconer, D.S., 1989. Introduction to Quantitative Genetics, 3rd edn. Longman Scientific and Technical, Essex, England.

Fogelson, D.L., Nuechterlein, K.H., Asarnow, R.F., Subotnik, K.L., Talovic, S.A., 1991. Interrater reliability of the structured clinical interview for DSM-III-R, Axis II: schizophrenia spectrum and affective spectrum disorders. Psychiatry Research 39, 5563.

Fogelson, D.L., Nuechterlein, K.H., Asarnow, R.F., Payne, D.L., Subotnik, K.L., Giannini, C.A., 1999. The factor structure of schizophrenia spectrum personality disorders: signs and symptoms in relatives of psychotic patients from the UCLA family members study. Psychiatry Research 87, 137-146.

Green, M.F., Nuechterlein, K.H., Breitmeyer, B., 1997. Backward masking performance in unaffected siblings of schizophrenic patients: evidence for a vulnerability indicator. Archives of General Psychiatry 54, 465-472.

Grove, W.M., Lebow, B.S., Clementz, B.A., Cerri, A., Medus, C., Iacono, W.G., 1991. Familial prevalence and coaggregation of schizotypy indicators: a multitrait family study. Journal of Abnormal Psychology 100, 115-121.

Kendler, K.S., 2000. Schizophrenia genetics. In: Sadock, B.J., Sadock, V.A. (Eds.), Comprehensive Textbook of Psychiatry, vol. 1. Lippincott, Philadelphia, pp. 1147-1159.

Kendler, K.S., Ochs, A.L., Gorman, A.M., Hewitt, J.K., Ross, D.E., Mirsky, A.F., 1991. The structure of schizotypy: a pilot multitrait twin study. Psychiatry Research 36, 19-36.

Kendler, K.S., McGuire, M., Gruenberg, A.M., Walsh, D., 1995. Schizotypal symptoms and signs in the Roscommon Family Study: their factor structure and familial relationship with psychotic and affective disorders. Archives of General Psychiatry 52, 296-303.

Kremen, W.S., Seidman, L.J., Pepple, J.R., Lyons, M.J., Tsuang, M.T., Faraone, S.V., 1994. Neuropsychological risk indicators for schizophrenia: a review of family studies. Schizophrenia Bulletin 20, $103-119$.

Lencz, T., Raine, A., Benishay, D.S., Mills, S., Bird, L., 1995. Neuropsychological abnormalities associated with schizotypal personality. In: Raine, A., Lencz, T., Mednick, S. (Eds.), Schizotypal Personality. Cambridge Univ. Press, Cambridge, pp. 289-328.

Maier, W., Falkai, P., Wagner, M., 1999. Schizophrenia-spectrum disorders. In: Maj, M., Sartorius, N. (Eds.), WPA Series: Evidence and Experience in Psychiatry. Schizophrenia, vol. 2. Wiley, Chichester, England, pp. 311-371.

Meehl, P., 1962. Schizotaxia, schizotypy, schizophrenia. American Psychology 17, 827-838.
Muthén, B., Muthén, L., 2001. MPLUS, Version 1.04. Muthén and Muthén, Venice, Calif.

Nuechterlein, K.H., 1991. Vigilance in schizophrenia and related disorders. In: Steinhauer, S.R., Gruzelier, J.H., Zubin, J. (Eds.), Handbook of Schizophrenia. Neuropsychology, Psychophysiology and Information Processing, vol. 5. Elsevier, Amsterdam, pp. 397-433.

Nuechterlein, K.H., Dawson, M.E., 1984. Information processing and attentional functioning in the developmental course of schizophrenic disorders. Schizophrenia Bulletin 10, 160-203.

Nuechterlein, K.H., Parasuraman, R., Jiang, Q., 1983. Visual sustained attention: image degradation produces rapid sensitivity decrement over time. Science 220, 327-329.

Nuechterlein, K.H., Edell, W.S., Norris, M., Dawson, M.E., 1986. Attentional vulnerability indicators, thought disorder, and negative symptoms. Schizophrenia Bulletin 12, 408-426.

Nuechterlein, K.H., Dawson, M.E., Gitlin, M., Ventura, J., Goldstein, M.J., Snyder, K.S., Yee, C.M., Mintz, J., 1992. Developmental processes in schizophrenic disorders: longitudinal studies of vulnerability and stress. Schizophrenia Bulletin 18, $387-425$.

Nuechterlein, K.H., Asarnow, R.F., Subotnik, K.L., Fogelson, D.L., Ventura, J., Torquato, R.D., Dawson, M.E., 1998. Neurocognitive vulnerability factors for schizophrenia: convergence across genetic risk studies and longitudinal trait/state studies. In: Lenzenweger, M.F., Dworkin, R.H. (Eds.), Origins and Development of Schizophrenia: Advances in Experimental Psychopathology. American Psychological Association, Washington, DC, pp. 299-327.

Rado, S., 1953. Dynamics and classification of disordered behavior. American Journal of Psychiatry 110, 406-416.

Raine, A., Reynolds, C., Lencz, T., Scerbo, A., Triphon, N., Kim, D., 1994. Cognitive-perceptual, interpersonal, and disorganized features of schizotypal personality. Schizophrenia Bulletin 20, 191-201.

Reitan, R.M., 1958. Validity of the trail making test as an indicator of organic brain damage. Perceptual and Motor Skills 8, 271276.

Spitzer, R.L., Endicott, J., Gibbon, M., 1979. Crossing the border into borderline personality and borderline schizophrenia. Archives of General Psychiatry 36, 17-24.

Spitzer, R.L., Williams, J.B.W., Gibbon, M., First, M., 1990. Structured Clinical Interview for DSM-III-R-Personality Disorders (SCID-II, Version 1.0). American Psychiatric Press, Washington, DC.

Strandburg, R.J., Marsh, J.T., Brown, W.S., Asarnow, R.F., Guthrie, D., Higa, J., 1991. Reduced attention-related negative potentials in schizophrenic children. Electroencephalography and Clinical Neurophysiology 79, 291-307.

Subotnik, K.L., Nuechterlein, K.H., Asarnow, R.F., Fogelson, D.L., Goldstein, M.J., Talovic, S.A., 1997. Depressive symptoms in the early course of schizophrenia: relationship to familial psychiatric illness. American Journal of Psychiatry 154, 1551-1556.

Venables, P.H., 1995. Schizotypal status as a developmental stage in studies of risk for schizophrenia. In: Raine, A., Lencz, T., Mednick, S.A. (Eds.), Schizotypal Personality. Cambridge Univ. Press, Cambridge, pp. 107-131. 
Venables, P.H., Bailes, K., 1994. The structure of schizotypy, its relationship to subdiagnoses of schizophrenia and to sex and age. British Journal of Clinical Psychology 33, 277-294.

Vollema, M.G., Postma, B., in press. Neurocognitive correlates of schizotypy in first-degree relatives of schizophrenia patients. Schizophrenia Bulletin.
Vollema, M.G., van den Bosch, R.J., 1995. The multidimensionality of schizotypy. Schizophrenia Bulletin 21, 19-31.

Wechsler, D., 1955. Manual for the Wechsler Adult Intelligence Scale. The Psychological Corporation, New York. 